\title{
Web Embedded Server for Remote Access Monitoring and Controlling
}

\author{
Ms. Aiysha I. Khairatkhan ${ }^{1}$, Prof. Sumalatha D. Bandari ${ }^{2}$ \\ Student, Department of CSE, AGTI'S Dr. Daulatrao Aher College of Engineering, Karad, India ${ }^{1}$ \\ Professor, Department of CSE, AGTI'S Dr. Daulatrao Aher College of Engineering, Karad, India ${ }^{2}$
}

\begin{abstract}
Embedded Web System is a smart system designed to perform one or a few dedicated functions, often with real-time computing constraints. An embedded system is usually embedded as part of a complete device including hardware and mechanical parts. Instead of PC oriented servers, the ARM processor based servers are becoming trend of today's market. Due to its reduced cost and size high performance is achieved using ARM processor along with Ethernet module as Embedded Web Server. Some Embedded Systems are mass-produced, benefiting from economies of scale. Idea is utilized for monitoring and controlling maximum number of home appliances as well as industry devices. Since the Embedded System is dedicated to specific tasks, for industry automation, instrumentation and household devices control etc, this is an optimized solution by engineer, reducing the size and cost of the product, or increasing the reliability and performance. System home page can be accessed using web browser. For sensing the temperature, light and humidity different sensors installed at working place. The different electronic devices are connected to ARM through UART ports. The data from these electronic devices are stored in ARM micro controllers through RS-232 serial bus communication.
\end{abstract}

Keywords: ARM, DACs, Embedded Web Server (EWS), Monitoring.

\section{INTRODUCTION}

Web server is nothing but it is a one kind of host machines (a web site) that provides reliable services for only requesting clients. Web server should consist of intelligent operating system, with faster processor speed, special purpose of hardware, large amount of memory storage, running applications and few web pages etc. Microcontroller or ARM processor is an embedded network that creates a way for easy controlled activities of any device from any remote location. Such servers designed using very low resource usage, are highly reliable, portable and secure systems. The EWS provides an Ethernet connection between the IGC100 and a network. The EWS allows monitoring and control of the IGC100 (and vacuum system) from a local network or the world-wide web.

Such Web Servers are developed using specialized or advanced computers. They use different kind of operating systems such as UNIX, Linux, and Windows, NT etc.

For example, handheld computers share some elements with embedded systems such as the operating systems and microprocessors.

The web server gives us the reliability and flexibility of monitoring and controlling the electronic devices/ appliances from every nook and corner of the universal world. Such web server systems apply typical client-server architecture where the client request to the server and accesses the server through the LAN router and the global Internet. Multiple clients send the request to the server. Firstly that request is processed by the router to connect to the Internet. Then the web processes the request made and finally connects to the desired web server. At last the Requested data is sent to the client through web server. An Embedded Web Server ARM processor that includes software and application code to monitor and control the systems.

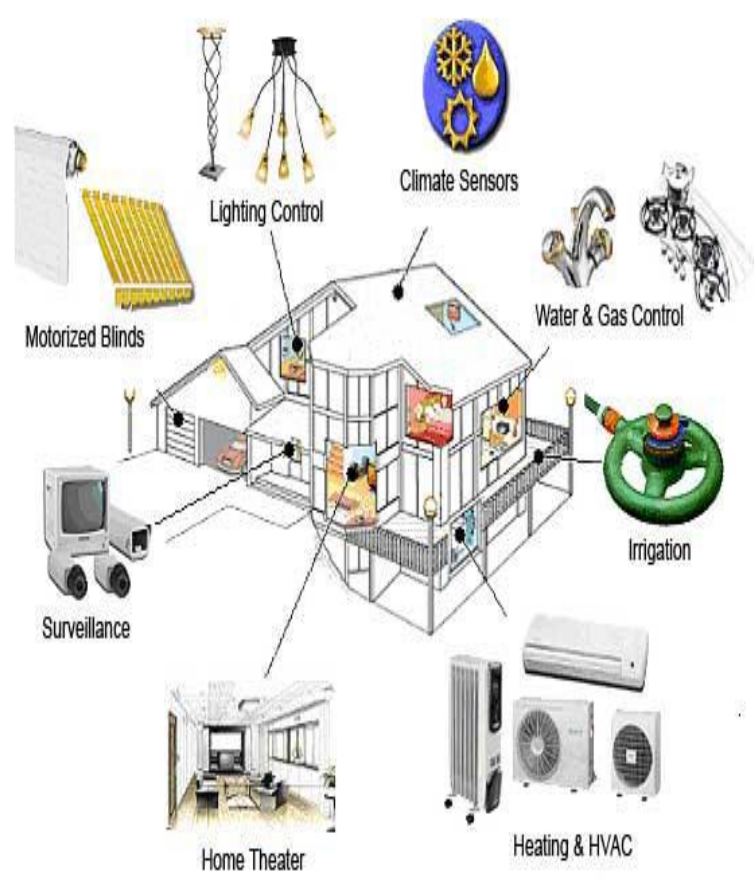

Fig.1: Different Devices Connected For Automation. 


\section{IARJSET}

\section{ARCHITECTURE OF EWS}

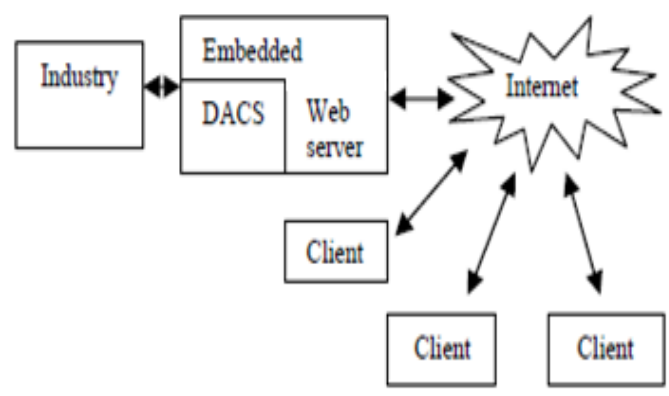

Fig. 2: Embedded Web-Server Architecture

The computers are growing rapidly with network technology; it has caused the technical revolution with the industrial control systems. A web server can be in built (embedded) in a device to provide remote access. The embedded system stores the information of various appliances at home or industry machineries, either static or dynamic information, and the system provides the same information to the Web Browsers on demand [5]. This sort of Web Server is called an Embedded Web Server. That Web Server is more intelligent than any other it is of ARM processor with Ethernet Module it contains system software and applications for overall Automation, monitoring and controlling process. In the above Fig. 2 it shows the way how the data is taken with EWS on a single chip module. This single chip module is a single hardware which contains program with portable ARM processor. This ARM processor is the most responsible part for measuring signals and controlling the devices with remotely access, with the help of temperature, light, and humidity one can monitor or control the EWS. Those measurements can be done with (Digital to Analog Converters) DACs and the data are shared with clients through EWS. The system is completely designed to measure signals, conversion of signals, data base updates, through sending displaying the HTML Web pages to the clients and communicating with owner of the system using authentication password etc. Without alteration in messages the communication can be achieved in correct manner.

\section{III.ETHERNET MODULE}

The Ethernet specification (IEEE 802.3) has evolved over the last number of years. The Ethernet module WIZ810MJ is used to address higher transmission rates and new functionality. This network module includes W5100 (TCP/IP hardwired chip, include PHY), MAG-JACK (RJ45 with X'FMR) with other glue logics. It can be used as a component and no effort is required to interface W5100 and transformer.

WIZ810MJ consists of W5100 and MAG-JACK. The WIZ810MJ is an ideal option for users who want to develop their Internet enabling systems rapidly.

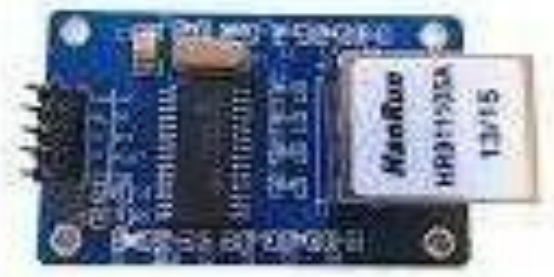

Fig. 3: Ethernet Module

A. Ethernet Specifications

- Network: 2 x shielded Neutrik Ethercon connector 2 x RJ45

- TCP/IP, MAC protocol layer : W5100

- Supported Protocols: DMX512 (1986 \& 1990),

- DMX512-A,

- DMX Timing: Frame rate : 10/40fps / Break:

- $176-352 \mu$

- Supported Protocols: Art-Net TM, scan ANSI E1.31

- Port Speed: 10/100Mbps

- Port Sensing: Auto-negotiation, MDI /

- MDIX

- RJ-45 Connector RDA - 125BAG1A

- Physical layer : Included in W5100

- Connector : MAG-JACK

- Input Voltage : $3.3 \mathrm{~V}$ Internal Operation and $5 \mathrm{~V}$ Tolerant I/Os

- Power Consumption : 10/100 base T : Max 185mA $(3.3 \mathrm{~V})$

\section{B. Features}

- Supports 10/100 Base TX

- DMX Port Direction: Input or output

- (configurable)

- DMX Port Isolation: Optic and galvanic isolation

- per port

- Supports half/full duplex operation

- IEEE 802.3/802.3u Complaints

- Operates 3.3V with 5V I/O signal tolerance

- Supports network status indicator LEDs

- Includes Hardware Internet protocols

- Includes Hardware Ethernet protocols: DLC, MAC

- Supports 4 independent connections simultaneously

- Supports Socket API for easy application programming

- Interfaces with Two $2.0 \mathrm{~mm}$ pitch $2 * 14$ header pin

\section{Advantages}

- Bandwidth Efficiency is more than other

- Finer Granularity is achieved

- Bandwidth protection

- Topologies are flexible

- Ubiquitous Connectivity

- Operational Simplicity

- Lower Cost

- Future Proof Investment Protection 
IARJSET

\section{Disadvantages}

- Difficult to change

- Fault Intolerant

- Difficult troubleshooting

- Specialized cable

\section{IV.WORKING OF THE SYSTEM}

- The collected information by sensor is given as inputs to ARM controller.

- It processes on digital or analog signals and then parameter values or readings are stored in the memory.

- On the basis of the stored values the status of working device are obtained.

- To change the status of devices as per our requirements relay can be operated. Either ON or OFF.

- Some standard values are already defined.

- After comparing the standard values and the tested values of the parameters, further status of devices is decided.

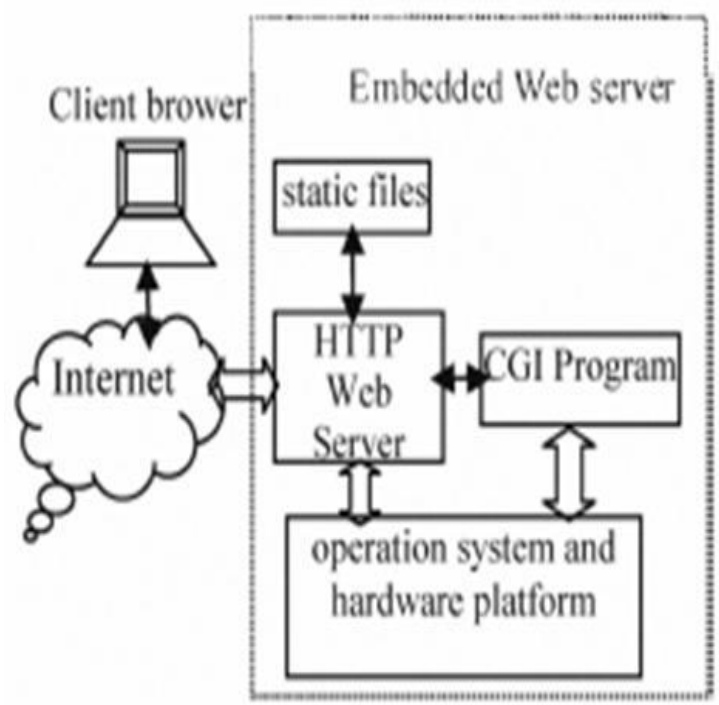

Fig.4: Embedded Web Server (EWS)

- For controlling the devices using web browser, owner of the system has to access the webpage and change the settings.

- Whole system developed code is written in C.

- That code will be transferred to processor using serial port. It is interfaced using driver/receiver interface.

- Microcontroller is responsible for processing the sense data and the feedback status is continuously provided to the website.

- Ethernet module transfers web pages to the client.

- Web page code is designed in HTML and uploaded on server using Ethernet module and TCP/IP address.

\section{FUTURE SCOPE}

The implementation of the system control application has been made in $\mathrm{C}$ language reducing its complexity. In addition, other master controls can be added to control more devices with their necessary communication routes if they have to control the new slaves.

The chosen interface effectively accomplishes its objectives while its use is easy contributing to an acceptance of the system by its users.

\section{CONCLUSION}

The EWS is a good media to attach device to internet. With the rapid development computers in the field of industrial process control and there is wide range of applications of network, intellectual intelligence, digital distributed control system, it is necessary to make a higher demand of the data accuracy and reliability of the control system for monitoring devices. There are lots of benefits in applying EWS technology to industrial as well as home automation control systems field. This system is built with low cost and is easy to install.

This embedded ARM system can adapt strict requirements of temperature, humidity, and light parameters in such a way that it is utilized to control and automate all present systems. Existing system is taking care of its function, reliability, power consumption, and remote access and can be expanded to suite some additional features. The designed system can be expanded for controlling more number of devices.

\section{ACKNOWLEDGMENT}

I am giving a sincere thanks to my Prof. S. D. Bandari madam for inspiring and sharing her wonderful thoughts regarding this subject with me. I am very thankful to our HOD of Computer Science and Engineering Department Prof. Ashish N. Patil Sir for helping me. A special thanks to everyone who has contributed for this research paper.

\section{REFERENCES}

[1] S. A. N. Sandeep, P. Malyadri "Embedded Web Server Based on DAC System Using ARM" International Journal of Engineering Research and Applications (IJERA) ISSN: 2248-9622, Vol. 2, Issue 4, July-August 2012.

[2] Deepak C. Karia, Vispi Adajania, Manisha Agrawal and Swapnil Dandekar "Embedded Web Server Application Based Automation and Monitoring System" Proceedings of 2011 International Conference on Signal Processing, Communication, Computing and Networking Technologies (ICSCCN 2011).

[3] Yanni Zhai, Xiaodong Cheng "Design of Smart Home Remote Monitoring System Based on Embedded System" 978-1-42449600-6/11//\$26.00 @2011 IEEE.

[4] Mo Guan Minghai Gu "Design and Implementation of an Embedded Web Server Based on ARM" School of Information Science \& Engineering Shenyang University of Technology Shenyang, China 978-1-4244-6055-7/10/\$26.00 @2010 IEEE.

[5] V. Billy Rakesh Roy1, Sanket Dessai1, S. G. Shiva Prasad Yadav "Design and Development of ARM Processor Based Web Server" International Journal of Recent Trends in Engineering, Vol. 1, No. 4, May 2009.

[6] A. R. Al-Ali, Member, IEEE \& M. AL-Rousan "Java-Based Home Automation System" IEEE Transactions on Consumer Electronics, Vol. 50, No. 2, MAY 2004. 
IARJSET

\section{BIOGRAPHIES}

Ms. Aiysha I. Khairatkhan is currently pursuing computer science engineering at Dr. Daulatrao Aher College of Engineering, Karad. Her area of interest includes Android Applications \& Web Designing.

Prof. Sumalatha D. Bandari received Diploma in Electronics \& Telecommunication Engineering from Government Polytechnic for Women, Nizamabad, Andhrapradesh in 2001. B. Tech degree from Jawaharlal Nehru Technological University, Hyderabad in Computer Science \& Information Technology in 2005, the M. Tech degree from Jawaharlal Nehru Technological University, Hyderabad in 2011. From 2006 to 2010 she worked as an Assistant Professor in Department of Information Technology of Indur Institute of Engineering \& Technology, Siddipet, Andhrapradesh. From 2011 to May 2012 she worked as an Assistant Professor in Department of Computer Science \& Engineering of Rajarambapu Institute of Technology, Sakharale, District Sangli, Maharashtra. Since June 2012 she is working as an Assistant Professor in Information Technology Department of Dr. Daulatrao Aher College of Engineering, Karad, District Satara, Maharashtra. Her area of interest includes Natural Language Processing, Machine Learning \& Cloud Computing. 